\title{
The generation of Ganymede's diffuse aurora through pitch angle scattering
}

\author{
Arvind K. Tripathi, Rajendra P. Singhal, and Onkar N. Singh II \\ Department of Physics, Indian Institute of Technology (Banaras Hindu University), Varanasi, 221005 (UP), India \\ Correspondence to: Arvind K. Tripathi (aktrip2001@yahoo.co.in)
}

Received: 14 August 2016 - Revised: 7 November 2016 - Accepted: 6 January 2017 - Published: 22 February 2017

\begin{abstract}
Diffuse auroral intensities of neutral atomic oxygen OI $\lambda 1356 \AA$ emission on Ganymede due to whistler mode waves are estimated. Pitch angle diffusion of magnetospheric electrons into the loss cone due to resonant wave-particle interaction of whistler mode waves is considered, and the resulting electron precipitation flux is calculated. The analytical yield spectrum approach is used for determining the energy deposition of electrons precipitating into the atmosphere of Ganymede. It is found that the intensities (4-30 $R$ ) calculated from the precipitation of magnetospheric electrons observed near Ganymede are inadequate to account for the observational intensities $(\leq 100 R)$. This is in agreement with the conclusions reached in previous works. Some acceleration mechanism is required to energize the magnetospheric electrons. In the present work we consider the heating and acceleration of magnetospheric electrons by electrostatic waves. Two particle distribution functions (Maxwellian and kappa distribution) are used to simulate heating and acceleration of electrons. Precipitation of a Maxwellian distribution of electrons can produce about $70 R$ intensities of OI $\lambda 1356 \AA$ emission for electron temperature of $150 \mathrm{eV}$. A kappa distribution can also yield a diffuse auroral intensity of similar magnitude for a characteristic energy of about $100 \mathrm{eV}$. The maximum contribution to the estimated intensity results from the dissociative excitation of $\mathrm{O}_{2}$. Contributions from the direct excitation of atomic oxygen and cascading in atomic oxygen are estimated to be only about 1 and $2 \%$ of the total calculated intensity, respectively. The findings of this work are relevant for the present JUNO and future JUICE missions to Jupiter. These missions will provide new data on electron densities, electron temperature and whistler mode wave amplitudes in the magnetosphere of Jupiter near Ganymede.
\end{abstract}

Keywords. Magnetospheric physics (energetic particles precipitating)

\section{Introduction}

Ganymede is a satellite of Jupiter and is the largest moon in our solar system. It has a radius of $2634 \mathrm{~km}$. It is larger than Mercury and Pluto and three-quarters the size of Mars. It orbits Jupiter at about 1.070 million $\mathrm{km}\left(\sim 15 R_{\mathrm{J}}, R_{\mathrm{J}}\right.$ is the radius of Jupiter, i.e., $71496 \mathrm{~km}$ ). Its orbit is very slightly eccentric and inclined to the Jovian equator, with the eccentricity and inclination changing quasiperiodically due to solar and planetary gravitational perturbations on a timescale of centuries. These orbital variations cause the axial tilt (the angle between rotational and orbital axes) to vary between 0 and $0.33^{\circ}$ (Susanna et al., 2002).

Several probes flying by or orbiting Jupiter have explored Ganymede more closely, including four flybys in the 1970s, and multiple passes in the 1990s to 2000s. Ganymede has been explored by the Pioneer 10 and 11 probes (Mead, 1974) and the Voyager 1 and 2 probes, and they returned information about the satellite (Scarf et al., 1979; Gurnett et al., 1979). The Galileo spacecraft entered orbit around Jupiter and made six close flybys to explore Ganymede (Gurnett et al., 1996). The spacecraft had a suite of instruments which included a magnetometer, energetic particle detector and plasma wave spectrometer. In addition, the Hubble Space Telescope (HST) also explored Ganymede and provided evidence for a tenuous oxygen atmosphere (exosphere) on Ganymede. The most recent close observations of Ganymede were made by New Horizons (Grundy et al., 2007). 
Data obtained from Galileo encounters have provided a large amount of new information about the moon. These include the discovery and verification of Ganymede's magnetic field, its magnetosphere, its trapped particle population, its interaction with the Jovian environment and plasma waves associated with Ganymede (Gurnett et al., 1996; Kivelson et al., 1996, 1997, 1998; Frank et al., 1997; Williams et al., 1997a, b, 1998; Williams and Mauk, 1997). The presence of a global magnetic field at Ganymede was inferred from the detection of the electromagnetic and electrostatic waves and radio emissions as the Galileo spacecraft approached Ganymede (Gurnett et al., 1996) and was later confirmed by Galileo's magnetometer data during closer flybys of the moon. These data have shown that Ganymede has an intrinsic magnetic field strong enough to generate a mini-magnetosphere (diameter $4-5 R_{\mathrm{G}}, R_{\mathrm{G}}$ is radius of Ganymede $=2631 \mathrm{~km}$ ) embedded within the Jovian magnetosphere (Kivelson et al., 1996, 1997, 1998). A model with a fixed Ganymede-centered dipole superimposed on the Jovian ambient field provided a good first-order match to the data and suggested equatorial and polar field strengths at Ganymede's surface of 750 and 1200 nT, respectively. These values are 6 to 10 times the $120 \mathrm{nT}$ ambient Jovian field strength at Ganymede's orbit. According to this model, magnetic field lines emanating from Ganymede's poles connect to Jupiter, whereas lines closer to Ganymede's equator intersect Ganymede's surface at both ends. The value of the moon's permanent magnetic moment is about $1.3 \times 10^{13} \mathrm{~T}$ $\mathrm{m}^{3}$, which is 3 times larger than the magnetic moment of Mercury. The data acquired during four close flybys of Galileo past Ganymede are consistent with a Ganymedecentered magnetic dipole tilted by $10^{\circ}$ from the spin axis towards $200^{\circ}$ Ganymede east longitude (Kivelson et al., 1996). The model of Ganymede's magnetic moment was further refined after the last two flybys: G28 and G29 (Kivelson et al., 1998). The current estimate is that the magnetic pole is tiled $4^{\circ}$ from the spin axis and points towards $156^{\circ} \mathrm{W}$ in the north and $336^{\circ} \mathrm{W}$ in the south. The interaction between the Ganymedian magnetosphere and Jovian plasma is similar in some respects to that of the solar wind and terrestrial magnetosphere.

No atmosphere was revealed by the Voyager data. Evidence for a tenuous oxygen atmosphere on Ganymede was found by the HST (Hall et al., 1998). The HST actually observed the airglow of atomic oxygen in the farultraviolet range at the wave lengths 130.4 and $135.6 \mathrm{~nm}$. Airglow emissions are characterized by the flux ratio $F$ $(1356 \AA) / F(1304 \AA)$ of roughly $1-2$, which suggests the dissociative electron impact excitation of $\mathrm{O}_{2}$. Inferred vertical column densities are in the range of $(1-10) \times 10^{14} \mathrm{~cm}^{-2}$. The observed double-peaked profile of the neutral atomic oxygen (OI) $1356 \AA$ feature indicated a non uniform spatial emission distribution that suggested two distinct and spatially confined emission regions, consistent with the satellite's north and south poles. Additional evidence of the oxy- gen atmosphere comes from the detection of various gases trapped in the ice on Ganymede (Calvin and Spencer, 1994; Spencer et al., 1995; Calvin et al., 1996). The evidence consisted of the spectroscopic detection of ozone $\left(\mathrm{O}_{3}\right)$, as well as absorption features that indicated the presence of $\mathrm{O}_{2}$.

The discovery of an intrinsic magnetic field associated with Ganymede was reinforced by the HST observations of atomic oxygen emission associated with the polar regions of the satellite (Hall et al., 1998) and by the subsequent ultraviolet images obtained in 1998 that revealed unambiguous polar auroral emission from Ganymede with a brightness of up to 300 Rayleigh $(R)$ in localized spots (Feldman et al., 2000). These images also show a background emission above the detection limit of $50 R$ but not exceeding $100 R$ across the rest of the disk of the satellite (Eviatar et al., 2001b). The oxygen emission is thought to be produced primarily by electron dissociative excitation of the molecular oxygen that dominates in Ganymede's tenuous atmosphere, although there is also likely a lesser contribution from electron excitation of the atomic oxygen component of the atmosphere. The OI emissions appear in both hemispheres, at latitudes above $\left|40^{\circ}\right|$, in accordance with Galileo magnetometer data that indicate the presence of an intrinsic magnetic field such that Jovian magnetic field lines are linked to the surface of Ganymede only at high latitudes. Both the brightness and relative north-south intensity of emission vary considerably during the $5.5 \mathrm{~h}$ of observation, presumably because of the changing Jovian plasma environment at Ganymede.

McGrath et al. (2013) present the ultraviolet images of Ganymede acquired with HST from 1998 to 2007, all of which show auroral emission from electron excited atomic oxygen. Ultraviolet emission at $1356 \AA$ is brightest at relatively high latitudes in the orbital trailing (upstream plasma) hemisphere and in an auroral oval that extends to as low as $\sim 10^{\circ} \mathrm{N}$ latitude in the orbital leading (downstream plasma) hemisphere. The overall emission morphology appears to be driven primarily by the strong Jovian magnetospheric plasma interaction with Ganymede. At any given longitude, the latitude of the brightest emission does not change significantly, but its brightness sometimes does. Ganymede's auroral emission is characterized by localized bright regions with a peak brightness of $\sim 100-400 R$. The peak emission intensity at the oval is the region that receives the maximum particle precipitation. The correspondence between the boundaries of the UV oval emission and electron precipitation is significant. The background emissions of an intensity of 50-100 $R$ are called the diffuse aurora at Ganymede. We study the diffuse emissions produced by Jovian magnetospheric electron precipitation into the atmosphere of Ganymede.

The diffuse aurora at Earth is explained as the result of the pitch angle diffusion of electrons into the loss cone and subsequent precipitation in the atmosphere by plasma waves. Two important wave modes which are being considered are electrostatic electron cyclotron harmonic (ECH) and whistler mode waves. In this paper, we are mainly concerned with dif- 
fusion process by whistler mode waves. The whistler mode is one of the modes of the propagation of electromagnetic waves below the electron cyclotron frequency, and the waves tend to propagate parallel to the ambient magnetic field. On 27 June 1996 the Galileo spacecraft made the first close flyby G1 of Ganymede. Intense plasma waves were detected over a region of space nearly 4 times Ganymede's diameter (Gurnett et al., 1996). The types of waves detected are whistler mode emissions, upper hybrid waves, electrostatic electron cyclotron waves and escaping radio emission. Electromagnetic and electrostatic plasma waves have also been observed in the middle (10-20 $\left.R_{\mathrm{J}}\right)$ magnetosphere of Jupiter (Scarf et al., 1979; Gurnett et al., 1979, 1996; Stone et al., 1992). Plasma waves in the middle magnetosphere are not limited to the region around Ganymede. Observations at Jupiter obtained by the Plasma Wave Instrument on board the Galileo spacecraft indicate that whistler mode chorus emissions with frequency-integrated power levels of $10^{-8} \mathrm{~V}^{2} \mathrm{~m}^{-2}$ or greater are observed commonly in the Jovian magnetosphere near the magnetic equator (Menietti et al., 2008) in the frequency range $400 \mathrm{~Hz}<f<8 \mathrm{kHz}$. The emissions are relatively narrow-banded.

The planetary magnetospheres produce various plasma instabilities which lead to the emission of plasma waves propagating in various modes. Most of these instabilities are due to anisotropic electron distribution, such as beam, a loss-cone feature and temperature anisotropy. If the pitch angle distribution is anisotropic with more energy perpendicular than parallel to the magnetic field, a particle distribution is unstable. There is free energy to cause wave instability. Electron pitch angle anisotropy produced by the atmospheric loss cone may produce electrostatic ECH and whistler mode instability in a magnetosphere. The loss-cone anisotropy is produced when particles moving within a cone of directions along the magnetic field strike the planetary atmospheric surface and are lost from the system (Kennel and Petschek, 1966). Both ECH and whistler mode waves can cause pitch angle diffusion of electrons which results in particle precipitation into the atmosphere producing a diffuse aurora.

In addition to causing pitch angle diffusion, electrostatic waves can also heat and accelerate the ambient electrons (Swift, 1970). This can produce a non-Maxwellian and suprathermal tail of energetic electrons in the magnetosphere at the magnetic equator. Such non-thermal distributions, with overabundances of fast particles, can be better fitted for superthermal velocities by generalized Lorentzian or kappa distributions. In this distribution function, characterization is done by real values of "spectral index $(\kappa)$ ", which assumes different shapes (Summers and Thorne, 1991; Summers et al., 1994). At high velocities, the distribution has an inverse power law tail in energy with the exponent $(\kappa+1)$.

In a recent work Singhal et al. (2016) have studied the diffuse aurora on Ganymede due to pitch angle diffusion of electrons by ECH waves. In the present study we have extended this work and calculated the diffuse auroral intensi- ties from pitch angle diffusion of magnetospheric electrons by whistler mode waves. In Sect. 2 we present the details on the method of calculations. The potential results of the study are discussed in Sect. 3, and finally the concluding remarks of the present work have been provided in Sect. 4 .

\section{Calculation details}

\subsection{Loss cone at Ganymede}

Due to the unique location of Ganymede in the Jovian magnetosphere, the field lines emanating from Ganymede are connected to Jupiter above a latitude of about $|\lambda|>40^{\circ}$. There are two mirror points on this field line: one near Ganymede and the other near Jupiter. The minimum magnetic field on this field line defines the magnetic equator. The Ganymede side loss cone can be calculated from the conservation of the first adiabatic invariant, i.e.,

$\operatorname{Sin}^{2} \alpha / \boldsymbol{B}=$ Constant

where $\alpha$ is the electron pitch angle and $\boldsymbol{B}$ is the magnetic field. Using Eq. (1) we obtain

$\operatorname{Sin}^{2} \alpha_{\mathrm{LC}}=\boldsymbol{B}_{\mathrm{eq}} / \boldsymbol{B}_{\mathrm{A}}$,

where $\alpha_{\mathrm{LC}}$ is the loss-cone angle, $\boldsymbol{B}_{\text {eq }}$ is the field at the magnetic equator and $\boldsymbol{B}_{\mathrm{A}}$ is the field at the top of the atmosphere of Ganymede. The density of $\mathrm{O}_{2}$ becomes $1 / e$ $(e=2.71828 \ldots)$ of its value at the surface at a radial distance of $1.005 R_{\mathrm{G}}$ (Eviatar et al., 2001b). The top of the atmosphere is assumed at $1.005 R_{\mathrm{G}}$. For calculating the $\boldsymbol{B}$ fields, we trace the field line connecting Ganymede and Jupiter using the equations

$1 / r \mathrm{~d} r / \mathrm{d} \theta=\boldsymbol{B}_{\mathrm{r}} / \boldsymbol{B}_{\theta}$ and $\sin \theta \mathrm{d} \varphi / \mathrm{d} \theta=\boldsymbol{B}_{\varphi} / \boldsymbol{B}_{\theta}$,

where $r$ is the distance from center of Jupiter, $\theta$ and $\varphi$ are colatitudes and longitude measured in a Jupiter-centered spherical coordinate system, and $\boldsymbol{B}_{\mathrm{r}}, \boldsymbol{B}_{\theta}$ and $\boldsymbol{B}_{\varphi}$ are the field components. The magnetic field of the Ganymede-Jupiter system is calculated, assuming the static superposition, from (Tripathi et al., 2013, 2014)

$\boldsymbol{B}=-\nabla V+\boldsymbol{b}+\boldsymbol{b}_{\mathrm{G}}$

The magnetic field of Jupiter is a sum of contributions from internal and external sources. The internal field is derivable from a scalar potential $V$. The VIP4 model (Connerney et al., 1998) is used, in which $V$ is expressed as a spherical harmonic expansion to degree and order 4 . The external field $b$ is due to a thin disc-shaped azimuthal current sheet. It is calculated using the analytical expressions given by Connerney et al. (1981, 1982) and Acuna et al. (1983). The magnetic field of Ganymede $\boldsymbol{b}_{\mathrm{G}}$ is modeled by a Ganymede-centered dipole (Kivelson et al., 1998). Loss-cone angles calculated in the present work are $\alpha_{\mathrm{LC}} \approx 10.8-13.6^{\circ}$. It may be noted that 
the magnetic field model of Ganymede given by Kivelson et al. (1996) has been used in the work of Tripathi et al. (2013). In this work we have used the refined model suggested by Kivelson et al. (1998).

\subsection{Whistler mode wave growth rate at the magnetic equator}

The temporal growth rate for whistler mode waves is calculated using the appropriate dispersion relation. For parallel propagating $R$ mode, the dispersion relation is written as (Kennel and Petschek, 1966)

$$
\begin{aligned}
& D(\boldsymbol{k}, \omega)=1-\frac{c^{2} \boldsymbol{k}^{2}}{\omega^{2}}-\pi \sum_{\alpha} \frac{\omega_{p \alpha^{2}}}{\omega} \int_{0}^{\infty} v_{\perp}^{2} \mathrm{~d} v_{\perp} \int_{-\infty}^{+\infty} \mathrm{d} v_{\|} \\
& \times\left[\frac{\partial f_{\alpha}}{\partial v_{\perp}}-\frac{\boldsymbol{k}}{\omega}\left(v_{\|} \frac{\partial f_{\alpha}}{\partial v_{\perp}}-v_{\perp} \frac{\partial f_{\alpha}}{\partial v_{\|}}\right)\right] \frac{1}{\boldsymbol{k} v_{\|}-\omega+\Omega_{\alpha}}=0,
\end{aligned}
$$

where $\alpha$ denotes species, $\boldsymbol{k}$ is the parallel wave propagation vector, $\omega$ is complex frequency $\left(\omega=\omega_{\mathrm{r}}+i \gamma\right)$ and $v=v_{\|}+v_{\perp}$ is the velocity in which $v_{\|}$and $v_{\perp}$ are components parallel and perpendicular to the ambient magnetic field. $\omega_{\alpha}$ and $\Omega_{\alpha}$ are plasma and gyrofrequency, respectively. Parameter $f_{\alpha}$ is the electron distribution function. The distribution is a combination of Maxwellian (cold) and kappa loss-cone (hot) distributions $(\alpha=c, h)$. These are given by

$$
\begin{aligned}
& f_{\mathrm{M}}=\frac{1}{\pi^{3 / 2} v_{\mathrm{c}}{ }^{3}} \exp \left(-v_{\perp}^{2} / v_{\mathrm{c}}^{2}-v_{\|}^{2} / v_{\mathrm{c}}{ }^{2}\right) \\
& v_{\mathrm{c}}^{2}=\left(2 T_{\mathrm{c}} / m_{\mathrm{e}}\right) \\
& \text { and } f_{\kappa}=C_{1} \frac{1}{\left(1+\frac{E}{\kappa E_{o}}\right)^{(\kappa+1)}}(\sin \alpha)^{2 s}
\end{aligned}
$$

where $C_{1}$ is the normalization constant. Here, $\alpha$ is the pitch angle, $T_{\mathrm{c}}$ is the cold electron temperature, $n_{\mathrm{c}}$ is the cold electron density, $m_{\mathrm{e}}$ is the mass of electron, $s$ is the losscone index, $\kappa$ is the spectral index and $E_{o}$ is the characteristic energy. In the case of the Maxwellian distribution, $n_{\mathrm{c}}=12.5 \mathrm{~cm}^{-3}$ and $T_{\mathrm{c}}=18.6 \mathrm{eV}$; for the kappa loss-cone distribution, $\kappa=1.5$ and $E_{o}=500 \mathrm{eV}$ are taken. Hot electron density $n_{\mathrm{h}}=0.1 n_{\mathrm{c}}$, the index $s=0.5$ and ambient magnetic field $\boldsymbol{B}_{o}=55 \mathrm{nT}$ at the magnetic equator are considered. The parameters $n_{\mathrm{c}}$ and $T_{\mathrm{c}}$ are obtained at the magnetic equator using the analytical expressions presented by Divine and Garrett (1983). The parameters $\kappa, E_{o}$ and $n_{\mathrm{h}}$ are taken from the work of Paranicas et al. (1999). Details about solving the dispersion relation (Eq. 5) are described in Tripathi et al. (2014). The temporal growth rate profile for whistler mode waves, calculated in the present work, is presented in Fig. 1. The temporal growth rate profile is used to represent the whistler mode wave spectral intensity in the calculation of pitch angle diffusion coefficients.

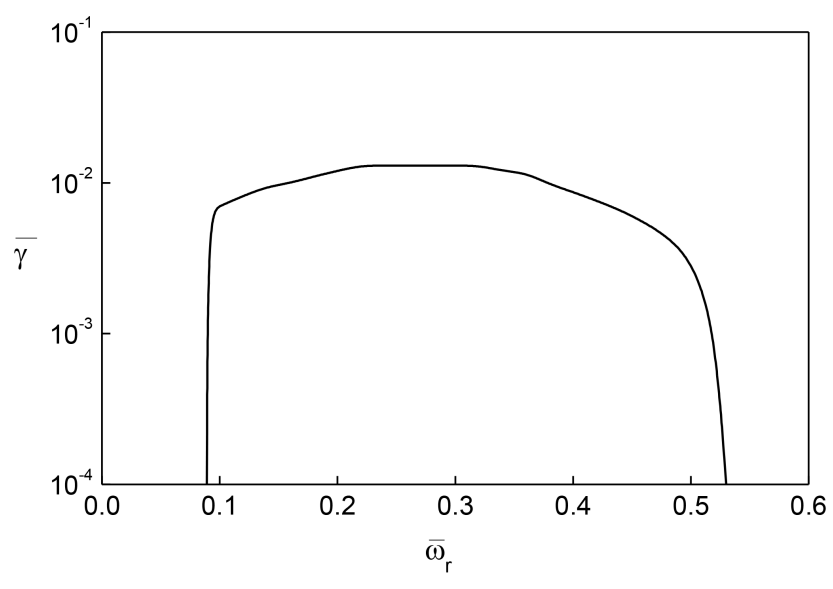

Figure 1. Normalized temporal growth rate $\bar{\gamma}\left(=\gamma / \Omega_{\mathrm{e}}\right)$ versus normalized real frequency $\bar{\omega}_{\mathrm{r}}\left(=\omega_{\mathrm{r}} / \Omega_{\mathrm{e}}\right)$ for whistler mode waves.

\subsection{Pitch angle diffusion coefficients}

The scattering of geomagnetically trapped particles is predominantly controlled by plasma waves that are Doppler shifted in frequency to some integral multiple of the particle cyclotron frequency, i.e.,

$\omega-k_{\|} v_{\|}=n \Omega_{\mathrm{e}} / \gamma, n=0, \pm 1, \pm 2, \ldots \ldots$,

where $\gamma=\left(1-v^{2} / c^{2}\right)^{1 / 2}$. The Landau $(n=0)$ resonance simply involves energy transfer between waves and particle. For the cyclotron $(n \neq 0)$ resonances, the diffusion occurs predominantly in pitch angle $(v=$ const).

We have calculated pitch angle diffusion coefficients $\left(D_{\alpha \alpha}\right)$ due to whistler mode waves using the expressions given by Lyons (1974) (also Singhal and Tripathi, 2006) under the high-density approximation $\left(\left(\omega_{\mathrm{pe}} / \Omega_{\mathrm{e}}\right)^{2} \gg \omega / \Omega_{\mathrm{e}}\right)$. This approximation simplifies the dispersion relation for whistler mode waves and the equations for the diffusion coefficients. We may write

$D_{\alpha \alpha}=\sum_{n=-\infty}^{\infty} \int_{0}^{x_{\max }} x \mathrm{~d} x D_{\alpha \alpha}^{n x}$,

where $x=\tan \eta$ and $\eta$ is the wave normal angle (the angle between $\boldsymbol{B}_{o}$ and the wave propagation vector $\left.\boldsymbol{k}\right) . D_{\alpha \alpha}{ }^{n x}$ is given by

$$
\begin{aligned}
D_{\alpha \alpha}{ }^{n x} & =\frac{\pi \cos ^{5} \eta \Omega_{\mathrm{e}}\left(-\sin ^{2} \alpha-n \Omega_{\mathrm{e}} / \omega_{k}\right)^{2} \cdot\left|\Phi_{n, k}\right|^{2}}{2 C_{1} \psi^{3 / 2}\left|1+n \Omega_{\mathrm{e}} / \omega_{k}\right|^{3} I\left(\omega_{k}\right)} \\
& \times f(\omega) g_{\omega}(x) \cdot\left(1-\left.\frac{1}{v_{\|}} \frac{\partial \omega_{k}}{\partial \boldsymbol{k}_{\|}}\right|_{x}\right)_{\mid\left(\frac{\omega_{k}}{\Omega_{\mathrm{e}}}\right)=\left(\frac{\omega_{k}}{\Omega_{\mathrm{e}}}\right)_{\mathrm{re}}}^{-1} \\
& \times \frac{\boldsymbol{B}_{\mathrm{wave}^{2}}}{\boldsymbol{B}_{o}{ }^{2}} \times \Omega_{\mathrm{e}} .
\end{aligned}
$$

$\alpha$ is the pitch angle, $v$ is electron speed and $\boldsymbol{B}_{\text {wave }}$ is the wave magnetic field. $f(\omega)$ is the wave spectral density and $g_{\omega}(x)$ 
gives the wave normal distribution. For $g_{\omega}(x)$ we use

$$
\begin{array}{ll}
g_{\omega}(x) \propto \exp \left(-x^{2}\right) & \text { for } x \leq 1, \\
0 & \text { for } x \geq 1 .
\end{array}
$$

It is assumed that the wave energy is proportional to the linear temporal growth rate. The constants of proportionality do not appear in the calculations of diffusion coefficients. The temporal growth rate profiles, therefore, represent the distribution of wave energy with frequency. For wave spectral density we have used the temporal growth rate profile shown in Fig. 1. In Eq. (10), $C_{1}=\int f(\bar{\omega}) \mathrm{d} \bar{\omega},\left(\bar{\omega}=\omega_{\mathrm{r}} / \Omega_{\mathrm{c}}\right)$ and

$$
\begin{aligned}
& \left(1-\left.\frac{1}{v_{\|}} \frac{\partial \omega_{k}}{\partial k_{\|}}\right|_{x}\right)=1-2 \psi\left\{\left[1+n \Omega_{\mathrm{e}} / \omega_{k}\right]\right. \\
& \times\left[2 \psi+2 \frac{\omega^{2}}{\Omega_{\mathrm{p}} \Omega_{\mathrm{e}}}-\frac{\omega^{2}}{\Omega_{\mathrm{p}}{ }^{2}}(1-M)^{2}\right. \\
& \left.\left.\times\left\{\left(1+x^{2}\right)\left(\psi-1+\frac{\omega^{2}}{\Omega_{\mathrm{p}} \Omega_{\mathrm{e}}}\right)+\frac{x^{2}}{2}\right\}^{-1}\right]\right\}^{-1}, \\
& \left|\Phi_{n, k}\right|^{2} \\
& =\left[\left(\frac{D}{\mu^{2}-S}\right)^{2}\left(\frac{\mu^{2} \sin ^{2} \eta-P}{\mu^{2}}\right)^{2}+\left(\frac{P \cos \eta}{\mu^{2}}\right)^{2}\right]^{-1} \\
& \times\left[\frac{\mu^{2} \sin ^{2} \eta-P}{2 \mu^{2}}\left(1+\frac{D}{\mu^{2}-S}\right) J_{n+1}+\frac{\mu^{2} \sin ^{2} \eta-P}{2 \mu^{2}}\right. \\
& \left.\times\left(1-\frac{D}{\mu^{2}-S}\right) J_{n-1}+\cot \alpha \sin \eta \cos \eta J_{n}\right]^{2} \text {, } \\
& I(\omega)=\int_{0}^{\infty} g_{\omega}(x) x\left\{\left(1+x^{2}\right) \psi\right\}^{-3 / 2} \\
& \left\{1+\frac{1}{\psi}\left[\frac{\omega^{2}}{\Omega_{\mathrm{p}} \Omega_{\mathrm{e}}}-\left\{\frac{1}{2} \frac{\omega^{2}}{\Omega_{\mathrm{p}}^{2}}(1-M)^{2}\right\}\right.\right. \\
& \left.\left.\times\left\{\left(1+x^{2}\right)\left(\psi-1+\frac{\omega^{2}}{\Omega_{\mathrm{p}} \Omega_{\mathrm{e}}}\right)+\frac{x^{2}}{2}\right\}^{-1}\right]\right\} \mathrm{d} x,
\end{aligned}
$$

where the argument of Bessel function $J_{n}=$ $J_{n}\left(x \tan \alpha\left(-\frac{\omega_{k}}{\Omega_{\mathrm{e}}}-n\right)\right)$

$\mu^{2}=\frac{\omega_{\mathrm{pe}}{ }^{2}}{\Omega_{\mathrm{e}}{ }^{2}} \frac{1+M}{M} \psi^{-1}$,

$M=m_{\mathrm{e}} / m_{\mathrm{p}}, m_{\mathrm{e}}$ and $m_{\mathrm{p}}$

are the mass of electrons and protons, respectively.

$$
\begin{aligned}
\psi & =1-\frac{\omega_{k}^{2}}{\Omega_{\mathrm{p}} \Omega_{\mathrm{e}}}-\frac{\sin ^{2} \eta}{2} \\
& +\left[\frac{\sin ^{4} \eta}{4}+\left(\frac{\omega_{k}}{\Omega_{\mathrm{p}}}\right)^{2}(1-M)^{2} \cos ^{2} \eta\right]^{1 / 2},
\end{aligned}
$$

$$
\begin{aligned}
P & =-\frac{\omega_{\mathrm{pe}}^{2}}{\Omega_{\mathrm{e}}^{2}} \frac{\Omega_{\mathrm{e}}^{2}}{\omega_{k}^{2}}(1+M), \\
S & =\frac{1}{2}(R+L), \\
D & =\frac{1}{2}(R-L), \\
R & R\}= \pm \frac{\omega_{\mathrm{pe}}^{2}}{\Omega_{\mathrm{e}}^{2}} \frac{\Omega_{\mathrm{e}}}{\omega_{k}}\left[\frac{1+M}{1-M \mp\left(\omega_{k} / \Omega_{\mathrm{e}}-\Omega_{\mathrm{p}} / \omega_{k}\right)}\right]
\end{aligned}
$$

Here, $\Omega_{\mathrm{p}}$ is proton gyrofrequency. Bounce-averaged diffusion coefficients are obtained from Lyons et al. (1972):

$$
\begin{aligned}
<D_{\alpha \alpha}> & =\frac{1}{\tau_{\mathrm{b}}} \int D_{\alpha \alpha}\left(\frac{\partial \alpha_{\mathrm{eq}}}{\partial \alpha}\right)^{2} \mathrm{~d} t \\
& =\frac{1}{\tau_{\mathrm{b}}} \int D_{\alpha \alpha}\left(\frac{\partial \alpha_{\mathrm{eq}}}{\partial \alpha}\right)^{2} \frac{\mathrm{d} s}{v \cos \alpha} .
\end{aligned}
$$

Using $(\mathrm{d} s)^{2}=(\mathrm{d} r)^{2}+r^{2}(\mathrm{~d} \theta)^{2}+r^{2} \sin ^{2} \theta(\mathrm{d} \varphi)^{2}$ and the conservation of first adiabatic invariant (Eq. 1), we can write

$$
\begin{aligned}
& <D_{\alpha \alpha}>=\frac{2}{v \tau_{\mathrm{b}}} \int_{\lambda_{\mathrm{G}}}^{\lambda_{\mathrm{J}}} \frac{D_{\alpha \alpha}(\alpha)}{\cos \alpha}\left(\frac{\tan \alpha_{\mathrm{eq}}}{\tan \alpha}\right)^{2} \\
& \times\left(r^{2}+\left(\frac{\partial r}{\partial \lambda}\right)^{2}+r^{2} \cos ^{2} \lambda\left(\frac{\partial \varphi}{\partial \lambda}\right)^{2}\right)^{1 / 2} \mathrm{~d} \lambda,
\end{aligned}
$$

where $r$ and $\theta, \varphi$ are the position on the field line connecting Ganymede with Jupiter $(\theta=\pi / 2-\lambda) . \lambda$ is latitude and $\lambda_{\mathrm{G}}$ and $\lambda_{\mathrm{J}}$ are mirror latitudes near Ganymede and Jupiter, respectively. $\tau_{\mathrm{b}}$ is the bounce period which is set equal to half the bounce period for Jupiter at $L=15$ (Orlova and Shprits, 2011). The variation in magnetic field and electron density along field lines is taken into account (Divine and Garrett, 1983; Eviatar et al., 2001a). Whistler mode waves are assumed to be confined within latitudes (measured from magnetic equator) $< \pm 13^{\circ}$ (Menietti et al., 2008). Pitch angle diffusion coefficients calculated in the current work are presented in Fig. 2 corresponding to two electron energies (200 eV and $2 \mathrm{keV}$ ) by using a wave magnetic field of $10 \mathrm{pT}$.

\subsection{Precipitation flux}

Pitch angle diffusion by whistler mode waves drives the magnetospheric electrons into the loss cone, thereby precipitating these electrons in the atmosphere of Ganymede. The differential flux of precipitation electrons as a function of energy and pitch angle is given by (Kennel and Petschek, 1966; Ni et al., 2012)

$J\left(E_{o}, \alpha\right)=J\left(E_{o}, \alpha_{\mathrm{LC}}\right) \frac{I_{o}\left(Z_{o} \alpha / \alpha_{\mathrm{LC}}\right)}{I_{o}\left(Z_{o}\right)}$,

where $Z_{o}=\sqrt{D_{\mathrm{SD}} /\left.\left\langle D_{\alpha \alpha}\right\rangle\right|_{\alpha_{\mathrm{LC}}}}$ is an energy-dependant parameter defining the diffusion strength near the loss cone. 


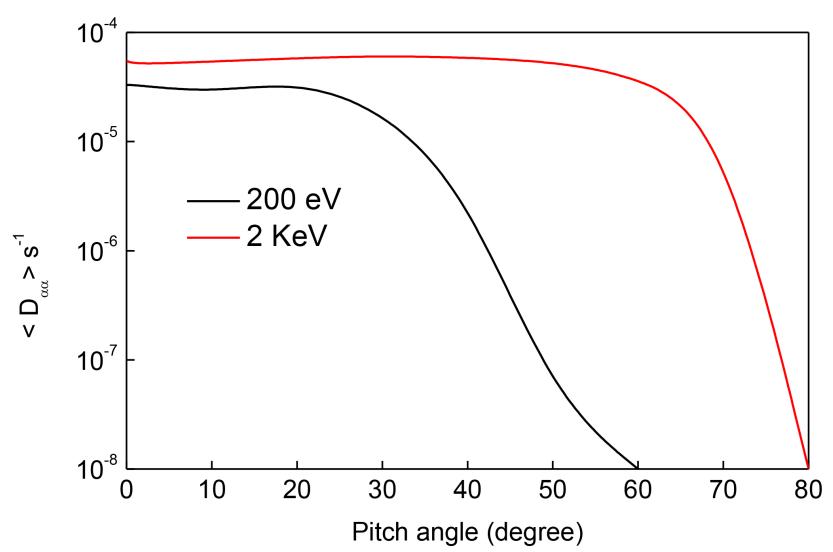

Figure 2. Bounce-averaged pitch angle diffusion coefficient versus pitch angle for whistler mode waves at two electron energies as indicated.

$I_{O}$ is the modified Bessel function of the first kind and $J$ $\left(E_{o}, \alpha_{\mathrm{LC}}\right)$ is the electron flux near the equatorial loss cone. $\left\langle D_{\alpha \alpha}\right\rangle_{\mathrm{LC}}$ is the bounce-averaged pitch angle diffusion coefficient at the edge of the loss cone. $D_{\mathrm{SD}}$ is the strong diffusion rate determined by (Kennel, 1969)

$D_{\mathrm{SD}}=\frac{2\left(\alpha_{\mathrm{LC}}\right)^{2}}{\tau_{B}}$.

Total precipitation flux $\Phi$ is determined by the differential flux of precipitating electrons inside the equatorial loss cone (e.g., Chang, 1983):

$\Phi=2 \pi \frac{\boldsymbol{B}_{A}}{\boldsymbol{B}_{\mathrm{eq}}} \int_{E_{1}}^{E_{2}} \int_{0}^{\alpha_{\mathrm{LC}}} J\left(E_{o}, \alpha\right) \cos \alpha \sin \alpha \mathrm{d} E_{o} \mathrm{~d} \alpha$.

Equatorial pitch angle $\alpha$ ( 0 to $\alpha_{\mathrm{LC}}$ ) maps to pitch angle $\theta_{o}(0$ to $\pi / 2$ ) at the top of the atmosphere. From the conservation of the first adiabatic invariant,

$\sin ^{2} \alpha / \boldsymbol{B}_{\mathrm{eq}}=\sin ^{2} \theta_{o} / \boldsymbol{B}_{\mathrm{A}} ;$

using Eq. (27), we can write Eq. (26) as

$\Phi=2 \pi \int_{E_{1}}^{E_{2}} \int_{0}^{\pi / 2} J\left(E_{o}, \alpha\left(\theta_{o}\right)\right) \sin \theta_{o} \cos \theta_{o} \mathrm{~d} E_{o} \mathrm{~d} \theta_{o}$,

where $\sin \alpha=\sin \theta_{o} \sin \alpha_{\mathrm{LC}} . E_{1}$ and $E_{2}$ are the lower and upper limit for integration over energy.

\subsection{Volume excitation rate}

For calculating the energy deposition of precipitating electrons in the atmosphere of Ganymede we have used the analytical yield approach (AYS) (Green and Singhal, 1979; Singhal et al., 1980; Singhal and Green, 1981) described

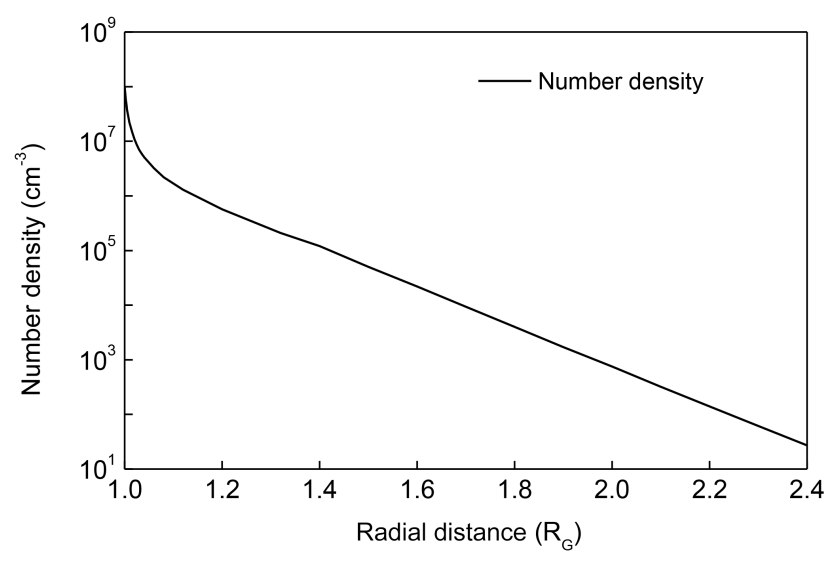

Figure 3. Variation in $\mathrm{O}_{2}$ number density.

in Appendix A. In this approach the volume excitation rate (VER) for exciting the $k$ th state in gas $i$ may be written as (cf. Eq. 28)

$$
\begin{aligned}
\mathrm{VER} & =2 \pi \int_{E_{1}}^{E_{2}} \mathrm{~d} E_{o} \int_{0}^{\pi / 2} \mathrm{~d} \theta_{o} \int_{W_{k i}}^{E_{o}} \mathrm{~d} E J\left(E_{o}, \alpha\left(\theta_{o}\right)\right) \\
& \times \sin \theta_{o} \cos \theta_{o} U\left(E, Z^{\prime}, E_{o}\right) \rho(Z) p_{k i}(E) .
\end{aligned}
$$

Here, $U\left(E, Z^{\prime}, E_{o}\right)$ is the three-dimensional AYS. $Z^{\prime}=$ $Z / \cos \theta_{o}, Z$ is the penetration depth in gram meters per square centimeters, $\rho$ is the atmospheric mass density and $p_{k i}$ is the excitation probability for exciting the $k$ th state in gas $i$. We use the model neutral $\mathrm{O}_{2}$ atmosphere computed by Eviatar et al. (2001b). The model $\mathrm{O}_{2}$ profile is presented in Fig. 3. We have also included atomic oxygen with a constant mixing ratio of $10 \%$. Vertical column density of the $\mathrm{O}_{2}$ profile is $2.9 \times 10^{14} \mathrm{~cm}^{-2}$.

In the present work we have calculated the OI $\lambda 1356 \AA$ diffuse auroral intensities. This wavelength arises from the atomic oxygen transition $\left(2 s^{2} 2 p^{4} 3 p \leftarrow 2 s^{2} 2 p^{3} 3 s^{5} s^{\circ}\right)$. We have considered three processes for the emission of this line. (1) Dissociative excitation of $\mathrm{O}_{2}$, (2) direct impact on atomic gas exciting the $\mathrm{O}\left({ }^{5} S^{\circ}\right)$ state and (3) direct impact on atomic $\mathrm{O}$ exciting the $\mathrm{O}\left({ }^{5} \mathrm{P}\right)$ state which cascades to O $\left({ }^{5} S^{\circ}\right)$. Probabilities of the excitation of the OI $\lambda 1356 \AA$ wavelength from these processes are

$$
\begin{aligned}
\text { (1) } P_{1} & =\frac{\sigma_{\mathrm{d}}}{0.1 \sigma_{1}+\sigma_{2}}, \\
\text { (2) } P_{2} & =\frac{0.1 \sigma\left({ }^{5} s^{0}\right)}{0.1 \sigma_{1}+\sigma_{2}} \\
\text { and } \quad \text { (3) } P_{3} & =\frac{0.1 \sigma\left({ }^{5} P\right)}{0.1 \sigma_{1}+\sigma_{2}} .
\end{aligned}
$$

Here, $\sigma_{1}$ and $\sigma_{2}$ are the total inelastic electron impact cross section for atomic $\mathrm{O}$ and molecular $\mathrm{O}_{2}$, respectively. $\sigma_{\mathrm{d}}$ is the cross section for dissociative excitation of $\mathrm{O}_{2}$. It is taken from the works of Wells et al. (1971), Erdman and Zipf (1987), 


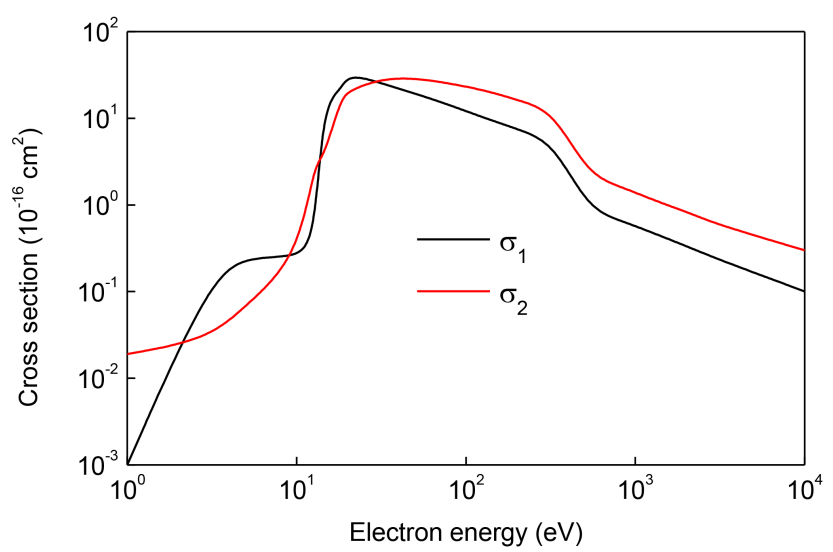

Figure 4. Total inelastic electron impact cross section versus electron energy for atomic oxygen $\left(\sigma_{1}\right)$ and molecular oxygen $\left(\sigma_{2}\right)$.

and Itikawa et al. (1989). Total inelastic electron impact cross sections $\sigma_{1}$ and $\sigma_{2}$ and cross sections for the direct excitation of atomic $\mathrm{O}$ to the states $\left({ }^{5} S^{\circ}\right)$ and $\left({ }^{5} P\right)$ are taken from the work of Jackman et al. (1977). Total inelastic cross sections $\sigma_{1}$ and $\sigma_{2}$ as a function of electron energy are presented in Fig. 4. The excitation cross sections $\sigma_{\mathrm{d}}, \sigma\left({ }^{5} S^{\circ}\right)$ and $\sigma\left({ }^{5} P\right)$ are plotted in Fig. 5.

For calculating the precipitation flux (Eq. 24), we require the flux $J\left(E_{o}, \alpha_{\mathrm{LC}}\right)$ at the edge of the loss cone. We have considered three flux profiles by assuming the pitch angle isotropy.

1. Flux of magnetospheric electrons observed near Ganymede (Frank et al., 1997; Paranicas et al., 1999).

$J\left(E_{o}\right)=a / E_{o}^{b}$ in units of $\left(\mathrm{cm}^{2} \mathrm{ssreV}\right)^{-1}$,

where $a=1.1 \times 10^{12}, b=3.52$ for the thermal component (9-100 eV), $a=1.6 \times 10^{7}, b=1.1$ for suprathermal electrons $(100 \mathrm{eV}-3 \mathrm{keV})$. Flux below $1 \mathrm{keV}$ has been extrapolated.

2. To simulate the heating of ambient electrons by electrostatic waves, we have considered the flux due to a Maxwellian distribution

$f_{\mathrm{M}}=\frac{n_{\mathrm{h}}}{\pi^{3 / 2} v_{\mathrm{h}}^{3}} \exp \left(-v^{2} / v_{\mathrm{h}}^{2}\right)$

where $v_{\mathrm{h}}=\left(2 T_{\mathrm{h}} / m\right)^{1 / 2}$.

3. Finally, to simulate the acceleration by electrostatic waves we have considered the flux due to a kappa distribution function:

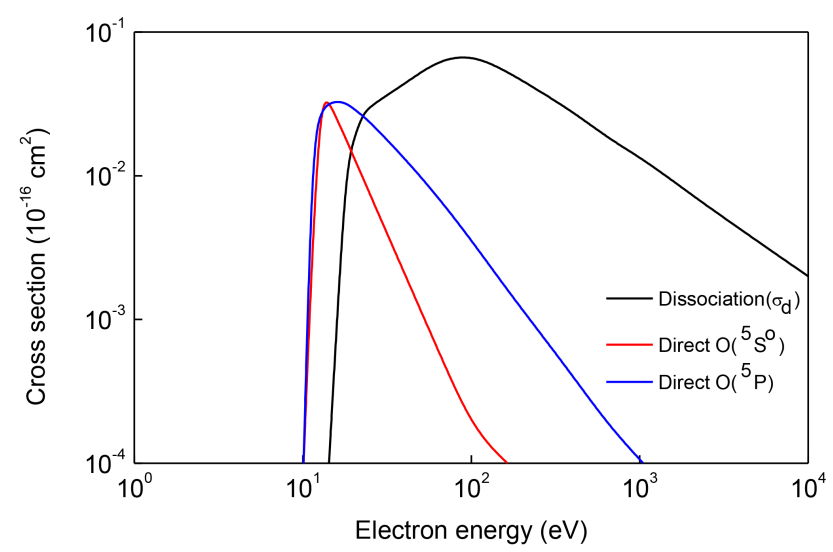

Figure 5. Electron impact excitation cross sections versus electron energy for dissociation $\left(\sigma_{\mathrm{d}}\right)$ and direct atomic $\mathrm{O}\left({ }^{5} S^{\circ}\right)$ and direct atomic $\mathrm{O}\left({ }^{5} P\right)$ excitations. Atomic $\mathrm{O}\left({ }^{5} P\right)$ cascades into $\mathrm{O}\left({ }^{5} S^{\circ}\right)$.

$$
\begin{aligned}
& f_{\kappa}=n_{\mathrm{h}}\left(\frac{m}{2 \pi E_{\mathrm{c}}}\right)^{3 / 2} \\
& \frac{\Gamma(\kappa+1)}{\kappa^{3 / 2} \Gamma(\kappa-1 / 2)} \frac{1}{\left(1+\frac{E}{\kappa E_{\mathrm{c}}}\right)^{\kappa+1}} .
\end{aligned}
$$

$E_{\mathrm{c}}$ is the characteristic energy. Two values of parameter $\kappa(\kappa=1$ and 2$)$ and $n_{\mathrm{h}}=12.5 \mathrm{~cm}^{-3}$ are used. Electron flux is obtained using

$J=2 E_{o} f / m^{2}$.

$m$ is the mass of electrons. Integrating the VER (Eq. 29) over altitude gives the intensity in Rayleigh ( $1 R=10^{6}$ photons $\left.\mathrm{cm}^{-2} \mathrm{~s}^{-1}\right)$. It is assumed that each excitation gives rise to a photon.

\section{Results and discussion}

In Fig. 1, it is observed that the calculated whistler mode wave growth rate is mainly confined within the normalized frequency range $\bar{\omega}\left(=\omega_{\mathrm{r}} / \Omega_{\mathrm{c}}\right) \approx 0.1-0.45$. This may be compared with the observations of whistler mode waves in the magnetosphere of Jupiter and in the vicinity of Ganymede. Observations of plasma waves made by Voyager 1 and 2 have been described by several authors (Scarf et al., 1979; Gurnett et al., 1979; Gurnett and Scarf 1983; Coroniti et al., 1984). Voyager 1 detected whistler mode emissions inside of $10 R_{\mathrm{J}}$ in the range of $\bar{\omega} \approx 0.3-0.42$. The same emissions detected by Voyager 2 extended out to $20 R_{\mathrm{J}}$ and beyond. Peak amplitudes of chorus waves received by Voyager were found to be about $0.26 \mathrm{mV} \mathrm{m}^{-1}(10 \mathrm{pT}$ amplitudes assuming parallel propagation). Whistler mode waves were also received near Io torus during the Ulysses-Jupiter encounter (Stone et al., 
1992 ) in the frequency range $\bar{\omega} \approx 0.04-0.2$. Recently, similar wave observations detected from the Galileo probe have been described by Menietti et al. (2008). The observations indicate that chorus emissions are observed commonly in the Jovian magnetosphere near the magnetic equator in the approximate radial range $9<r<13 R_{\mathrm{J}}$. These emissions exist in the frequency range from $\bar{\omega}=0.02$ up to, but seldom exceeding, about 0.46 . Wave magnetic field amplitude is found about $7 \mathrm{pT}$. Whistler mode waves have also been observed in the vicinity of Ganymede with a frequency of $\bar{\omega} \leq 0.5$ (Gurnett et al., 1996).

Figure 2 presents bounce-averaged pitch angle diffusion coefficients due to whistler mode waves. It is noticeable that the diffusion coefficients do not change much between two electron energies $(200 \mathrm{eV}$ and $2 \mathrm{keV})$ for pitch angle less than about $25^{\circ}$. At a higher pitch angle $\left(>50^{\circ}\right)$, the coefficients for an energy of $200 \mathrm{eV}$ are negligible, whereas for an energy of $2 \mathrm{keV}$ the coefficients extend up to about $80^{\circ}$. However, it is found that the diffusion coefficients are several orders of magnitude smaller below about $100 \mathrm{eV}$ and even become 0 for below $50 \mathrm{eV}$. The diffusion coefficients depend upon electron densities along the field line. Electron densities (and temperature) have been calculated using the analytical expressions provided by Divine and Garrett (1983) for the inner disc $\left(7.9<r<20 R_{\mathrm{J}}\right)$ in Jupiter's magnetosphere. These expressions are:

$N_{\mathrm{e}}=N \exp \left[-\left(\frac{r \lambda-Z_{o}}{H}\right)^{2}\right]$,

$k T=E_{o}-E_{1} \exp \left[\left(\frac{r \lambda-Z_{o}}{H}\right)^{2}\right]$.

$N$ is interpolated from Table 1 and

$H=(1.82-0.041 r) R_{\mathrm{J}}$

$Z_{o}=\left(\frac{7 r-16}{30} R_{\mathrm{J}}\right) \cos \left(\ell-\ell_{o}\right)$,

$E_{o}=100 \mathrm{eV}, E 1=85 \mathrm{eV}, \ell_{o}=21^{\circ}$.

Here, $r$ is the Jovicentric distance in $R_{\mathrm{J}} . \lambda$ is latitude (radian), and $\ell$ is longitude (degree in System III; 1965). The model is based primarily on in situ data returned by experiments on the Pioneer and Voyager spacecraft, supplemented by earth-based observations and theoretical considerations. The model represents the data typically to within a factor of $2^{ \pm 1}$ except where time variations, neglected in the model, are known to be significant. Further, we have made a highdensity approximation $\left(\left(\omega_{\mathrm{pe}} / \Omega_{\mathrm{e}}\right)^{2} \gg \omega / \Omega_{\mathrm{e}}\right)$ in the calculation of diffusion coefficients. In this work the ratio $\omega_{\mathrm{pe}}^{2} / \Omega_{\mathrm{e}}^{2}$ is 2-400. This range is due to variation in electron density and magnetic field along the field line connecting Ganymede to Jupiter. Glauert and Horne (2005) have presented a calculation of diffusion coefficients (PADIE code) for any ratio of $\beta\left(=\omega_{\mathrm{pe}} / \Omega_{\mathrm{e}}\right)$. It is found that the high-density approximation, at low electron energy $(<10 \mathrm{keV})$, agrees with the
Table 1. Equatorial parameter values for Jupiter's thermal charged particle populations (from Table 7 of Divine and Garrett, 1983).

\begin{tabular}{rr}
\hline Jovicentric distance & $\begin{array}{r}\text { Electron density } \\
r, R_{\mathrm{J}}\end{array}$ \\
\hline 7.9 & $\log N, \mathrm{~cm}^{-3}$ \\
\hline 10.0 & 2.25 \\
20.0 & 1.48 \\
& 0.20 \\
\hline
\end{tabular}

PADIE results for $\beta \geq 10$ but underestimates diffusion coefficients by 1 order of magnitude near the loss cone for $\beta \approx 1.5$. Resonant energy $\left(E_{\mathrm{M}}=B^{2} / 8 \pi n_{\mathrm{e}}\right)$ at the magnetic equator is found $\sim 600 \mathrm{eV}$ and increases up to $30 \mathrm{keV}$ along the field line connecting Ganymede to Jupiter.

The $\mathrm{O}_{2}$ number density presented in Fig. 3 is based on the Bates (1959) atmosphere model applied in the region $r \leq 1.38 R_{\mathrm{G}}$ and a coronal model in the exosphere region (Eviatar et al., 2001b). $\mathrm{O}_{2}$ surface density is $1 \times 10^{8} \mathrm{~cm}^{-3}$, and vertical column density is $2.9 \times 10^{14} \mathrm{~cm}^{-2}$. The atmosphere model is similar to that constructed by Feldman et al. (2000).

In Fig. 4 we present the electron impact total inelastic cross sections for atomic $\mathrm{O}$ and molecular $\mathrm{O}_{2}$. These cross sections have been calculated using the analytical expressions given by Jackman et al. (1977). For forbidden electron impact excitations, the cross section is written as

$\sigma=\left(\frac{q_{o} F}{W^{2}}\right)\left[1-\left(\frac{W}{E}\right)^{\alpha}\right]^{\beta}\left(\frac{W}{E}\right)^{\Omega}$.

The formula for allowed excitation is

$\sigma=\frac{\left(q_{o} F\right)\left[1-(W / E)^{\alpha}\right]^{\beta}}{E W} \ln \left(\frac{4 E C}{W}+e\right)$,

where $q_{o}=6.513 \times 10^{-14} \mathrm{eV}^{2} \mathrm{~cm}^{-2} . W$ is the parameter for the low-energy shape of the cross section and, in most cases is close to the energy loss. $F$ is the optical oscillator strength, and $e$ is the base of natural logarithm. $\alpha, \beta, \Omega$ and $C$ are adjustable parameters. The electron impact ionization cross sections are calculated from

$\sigma=\sigma_{o} A(E) \Gamma(E)$

$\left[\tan ^{-1}\left\{\frac{\left(T_{\mathrm{m}}(E)-T_{o}(E)\right)}{\Gamma(E)}\right\}+\tan ^{-1}\left\{\frac{T_{o}(E)}{\Gamma(E)}\right\}\right]$,

where

$$
\begin{aligned}
& A(E)=\left[\frac{K}{\left(E+k_{B}\right)}\right] \ln \left(\frac{E}{J}+J_{B}+\frac{J_{c}}{E}\right), \\
& \Gamma(E)=\Gamma_{\mathrm{S}} \frac{E}{\left(E+\Gamma_{B}\right)}, \\
& T_{o}(E)=T_{S}-\left[\frac{T_{A}}{\left(E+T_{B}\right)}\right],
\end{aligned}
$$


Table 2. Electron impact total ionization and total inelastic cross sections (in units of $10^{-16} \mathrm{~cm}^{2}$ ) for molecular oxygen and atomic oxygen gas (Jackman et al., 1977).

\begin{tabular}{lrr|rr}
\hline & $\mathrm{O}_{2}$ & & $\mathrm{O}$ \\
\hline Electron energy $(\mathrm{eV})$ & Total ionization & Total inelastic & Total ionization & Total inelastic \\
\hline 12.8 & 0.0 & 3.14 & 0.0 & 0.41 \\
20.3 & 20.2 & 21.2 & 32.2 & 33.1 \\
32.3 & 27.7 & 29.1 & 24.5 & 25.4 \\
51.4 & 27.6 & 29.2 & 18.4 & 19.0 \\
81.8 & 23.8 & 25.3 & 13.5 & 14.0 \\
130 & 19.3 & 20.6 & 9.86 & 10.2 \\
207 & 14.9 & 15.9 & 7.08 & 7.35 \\
330 & 11.0 & 11.7 & 5.02 & 5.21 \\
468 & 7.92 & 8.54 & 0.81 & 0.96 \\
525 & 1.62 & 2.20 & 0.76 & 0.89 \\
836 & 1.18 & 1.60 & 0.55 & 0.65 \\
1330 & 0.83 & 1.14 & 0.39 & 0.46 \\
2120 & 0.58 & 0.80 & 0.27 & 0.32 \\
3000 & 0.44 & 0.60 & 0.21 & 0.24 \\
\hline
\end{tabular}

Table 3. Calculated intensities of OI $1356 \AA$ emissions in Rayleigh due to magnetospheric electrons (Eq. 31).

\begin{tabular}{lcrrrr}
\hline $\begin{array}{l}\text { Electron } \\
\text { component }\end{array}$ & $1^{\mathrm{a}}$ & $2^{\mathrm{b}}$ & $3^{\mathrm{c}}$ & $4^{\mathrm{d}}$ & $5^{\mathrm{e}}$ \\
\hline Thermal & 6.1 & 0.002 & 0.005 & 0.009 & 16.0 \\
Suprathermal & 6.1 & 3.2 & 4.7 & 14.4 & 18.8 \\
\hline
\end{tabular}

${ }^{a}$ Using total magnetospheric electron flux; $\mathrm{O}_{2}$ column density

$2.9 \times 10^{14} \mathrm{~cm}^{2}$.

${ }^{\mathrm{b}}$ Using precipitation flux; whistler mode wave amplitude $10 \mathrm{pT} ; \mathrm{O}_{2}$ column density $2.9 \times 10^{14} \mathrm{~cm}^{2}$

${ }^{\mathrm{c}}$ Using precipitation flux; wave amplitude $20 \mathrm{pT} ; \mathrm{O}_{2}$ column density $2.9 \times 10^{14} \mathrm{~cm}^{2}$.

$\mathrm{d}$ Using precipitation flux; wave amplitude $20 \mathrm{pT} ; \mathrm{O}_{2}$ column density

$1.0 \times 10^{15} \mathrm{~cm}^{2}$.

${ }^{e}$ Using total magnetospheric electron flux; $\mathrm{O}_{2}$ column density

$1.0 \times 10^{15} \mathrm{~cm}^{2}$.

$T_{\mathrm{m}}(E)=\frac{\left(E-I_{\mathrm{i}}\right)}{2}$.

$\sigma_{o}=10^{-16} \mathrm{~cm}^{2}$ and $K, k_{B}, J, J_{B}, J_{c}, \Gamma_{S}, \Gamma_{B}, T_{S}, T_{A}$ and $T_{B}$ are adjustable parameters. $I_{\mathrm{i}}$ is the threshold energy for ionization. $E$ is electron energy. These are semiempirical cross sections based upon the experimental data. Various energydependent and other parameters appearing in Eqs. (37)-(39) are taken from Jackman et al. (1977). For atomic oxygen, 35 excited and Rydberg states and 3 ionizations have been included. For molecular oxygen, 12 excited and Rydberg states and 7 ionization states have been included in our calculations. For excitations of atomic oxygen presented in Fig. 5, calculations have been made using Eq. (37). The electron impact total ionization and total inelastic cross sections for molecular oxygen and atomic oxygen are also presented in Table 2.
In Table 3 we have presented the diffuse auroral intensities of OI $\lambda 1356 \AA$ emission resulting from the precipitation of magnetospheric electrons observed near Ganymede (Eq. 31). It is seen from Table 3 that the intensities due to precipitation of thermal electrons are negligibly small because diffusion coefficients are negligible for electron energies below $100 \mathrm{eV}$. Further, from the table it is also noticeable that total intensities for both thermal and superthermal electron components exist in the range of 3-35 $R$. These intensities are too small to account for the observational diffuse auroral intensities. The results of present calculations are in agreement with the conclusions reached in earlier works (Eviatar et al., 2001b). As discussed in the earlier works of Eviatar et al. (2001b), Lavrukhin and Alexeev (2015), and McGrath et al. (2013), some acceleration mechanism is required to energize the electrons. The processes that lead to the acceleration of electrons can be different in nature. In this paper, we consider the heating and acceleration by electrostatic waves observed in the magnetosphere of Jupiter and in the vicinity of Ganymede. For electrostatic waves, the wave normal is nearly perpendicular to the magnetic field lines. The component of phase velocity parallel to the field lines is high. An electron trapped in the wave may experience a substantial increase in velocity as it accelerates to keep up with the parallel component of the phase velocity. Particles could be accelerated by up to several times their initial velocity by this mechanism (Swift, 1970). The energetic electrons are subject to a frictional force in the ambient electron gas (Mantas, 1975):

$\mathrm{d} E / \mathrm{d} s=-\beta N_{\mathrm{e}} / E$,

where $\beta=2.59 \times 10^{-12}\left(\mathrm{eV}^{2} \mathrm{~cm}^{-2}\right), N_{\mathrm{e}}$ is the ambient electron density, $E$ is electron energy and $\mathrm{d} s$ is the element 


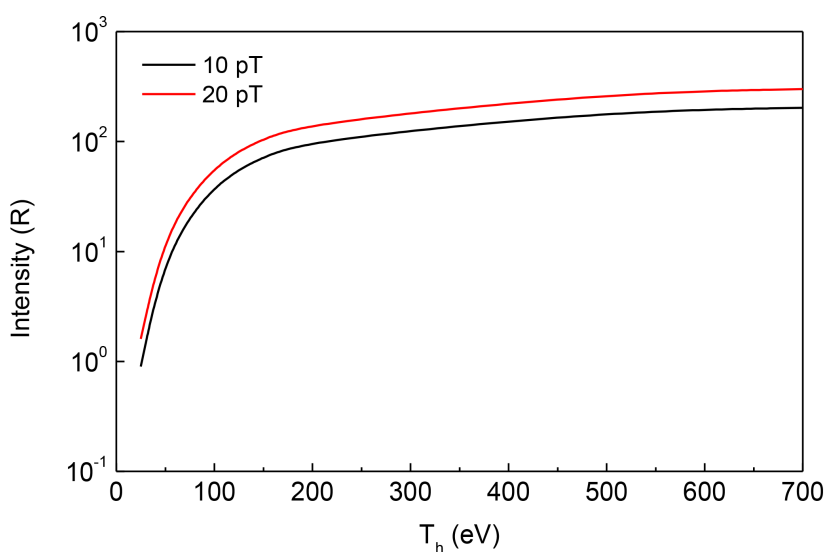

Figure 6. OI $1356 \AA$ intensity in Rayleigh $(R)$ versus electron temperature $\left(T_{\mathrm{h}}\right)$ for the Maxwellian distribution (Eq. 20). Whistler wave amplitude is indicated.

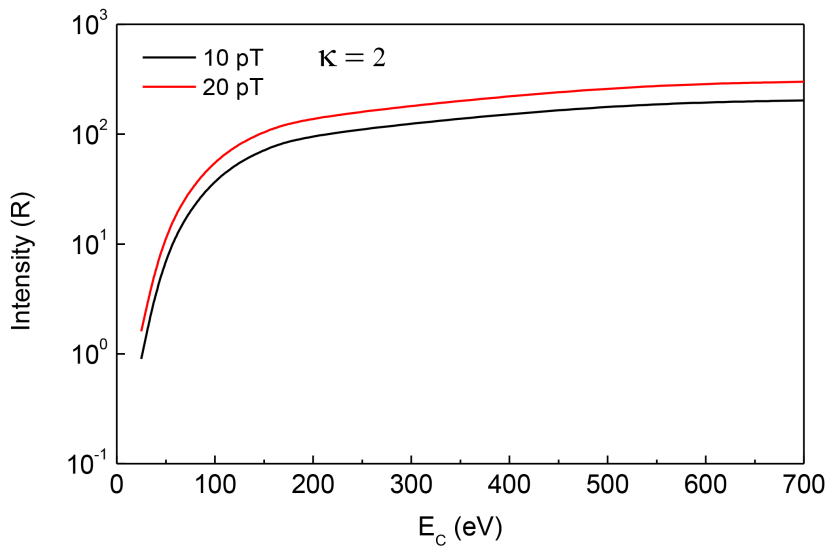

Figure 7. Intensity of OI 1356 Åemission in Rayleigh $(R)$ versus characteristic energy $\left(E_{\mathrm{c}}\right)$ for a kappa distribution with $\kappa=2$. Whistler wave amplitude is indicated.

of path length. The ambient electrons may be heated in this process. Electrostatic ECH waves have been observed in Jupiter's middle magnetosphere, with amplitudes of 1$5 \mathrm{mV} \mathrm{m}^{-1}$ (Scarf et al., 1979; Gurnett and Kurth, 1979). These waves have also been detected near Ganymede, confined to within a few degrees of the magnetic equator (Kurth et al., 1997; Gurnett et al., 1996). The electrostatic ECH wave turbulence may heat and accelerate the ambient. These electrons may precipitate into the atmosphere of Ganymede via pitch angle diffusion by whistler mode waves and may produce diffuse auroral emissions. We simulate the heating of ambient electrons using a Maxwellian distribution (Eq. 32). Calculated diffuse auroral intensities of OI $\lambda 1356 \AA$ emission are presented in Fig. 6 as a function of electron temperature $\left(T_{\mathrm{h}}\right)$. Calculations of this plot have been performed at two amplitudes of whistler mode wave: 10 and 20 pT. It is evident that intensities of about $70 R$ may be obtained from $10 \mathrm{pT}$ for a temperature of $150 \mathrm{eV}$. A higher temperature may

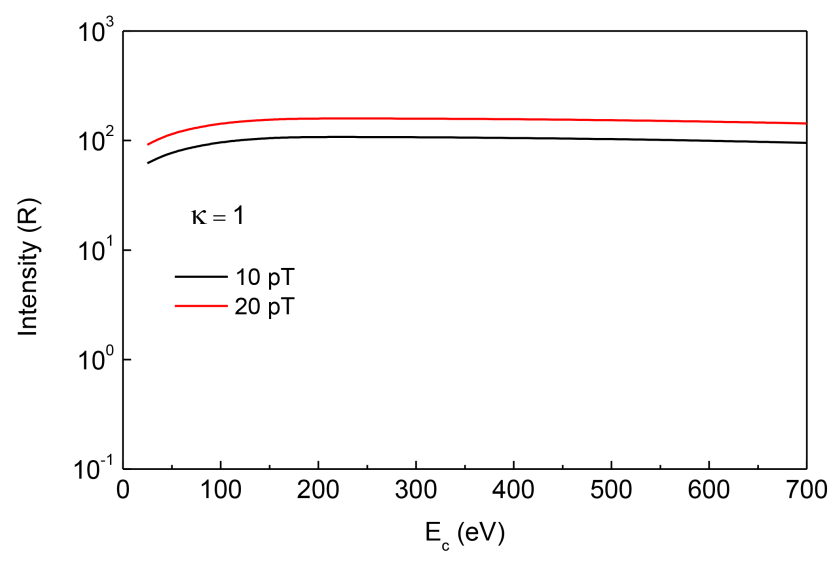

Figure 8. Same as in Fig. 7 for $\kappa=1$.

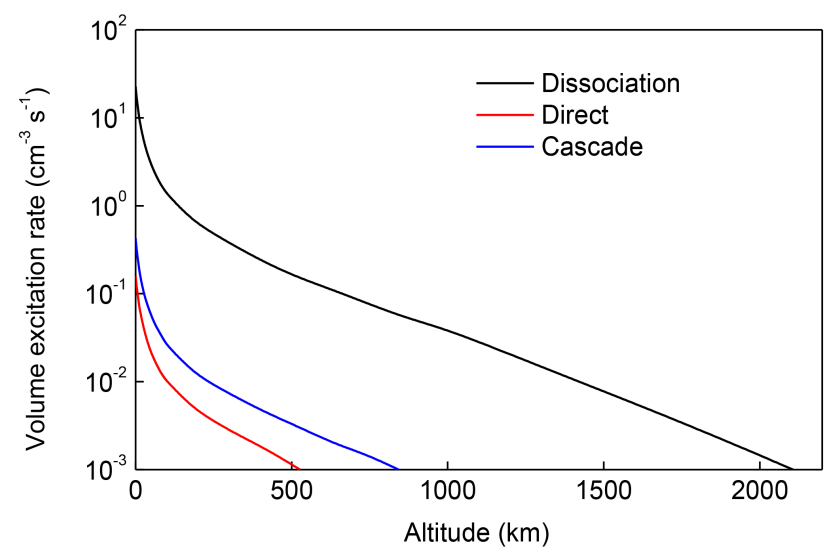

Figure 9. Volume excitation rate versus altitude for dissociation $\mathrm{O}_{2}$, direct $\mathrm{O}\left({ }^{5} S\right)$ and cascading $\mathrm{O}\left({ }^{5} P\right)$ excitations. A Maxwellian distribution with $T_{\mathrm{h}}=150 \mathrm{eV}$ is used, and whistler mode wave amplitude is $10 \mathrm{pT}$.

yield intensities of up to $200 R$. Further, the wave amplitude of $20 \mathrm{pT}$ may produce somewhat higher intensities as compared to values for $10 \mathrm{pT}$ at the same temperature. This is due to large pitch angle coefficients for $20 \mathrm{pT}$ since the diffusion coefficient scales as a square of the wave amplitude.

The acceleration of ambient electrons is simulated by a kappa distribution function (Eq. 33). Estimated diffuse auroral intensities are depicted in Figs. 7 and 8, corresponding to two values of $\kappa=2$ and $\kappa=1$, respectively. It is noted from Fig. 7 that the intensity $\approx 50-100 R$ may be obtained for a characteristic energy $E_{\mathrm{c}} \approx 100-150 \mathrm{eV}$. These values are higher in comparison to intensities obtained from the Maxwellian distribution (Fig. 6). In Fig. 8, intensities for $\kappa=1$ are somewhat higher (about $80 R$ for a characteristic energy $50 \mathrm{eV}$ ). This is due to the fact that more higher-energy electrons exist in the tail of a kappa distribution. It is also evident from Figs. 7 and 8 that intensities are flattened out for a characteristic energy $\geq 200 \mathrm{eV}$. Flattening is more in the case of $\kappa=1$ as compared to $\kappa=2$. This is probably due to 
the use of $3 \mathrm{keV}$ as the upper limit of integration over $E_{o}$ in Eq. (29). So, this may not capture higher energies in the tail of the distribution.

Finally, in Fig. 9 we show the altitude profile of the volume excitation rate for the Maxwellian distribution by considering the whistler wave amplitude $10 \mathrm{pT}$ and $T_{\mathrm{h}}=150 \mathrm{eV}$. It is noticeable that the electrons deposit their energy within about $200 \mathrm{~km}$ of the surface of Ganymede. Contribution from direct excitation of atomic $\mathrm{O}\left({ }^{5} S\right) 0.54 R$ is obtained, while cascading contributes only $1.4 R$. The maximum contribution of $72.9 R$ appears to be from dissociative excitation of $\mathrm{O}_{2}$.

\section{Conclusions}

Diffuse auroral intensities of OI $\lambda 1356 \AA$ emission have been calculated, resulting from pitch angle diffusion of magnetospheric electrons by whistler mode waves. Three cases are considered for the estimation of precipitation flux into the atmospheric loss cone. First, the intensities are estimated due to the precipitation of magnetospheric electrons observed near Ganymede. Next, the heating and acceleration of magnetospheric electrons by electrostatic waves is considered. Maxwellian and kappa distributions are used to simulate heating and acceleration, respectively. The AYS approach is used to calculate the energy deposition of electrons in the atmosphere of Ganymede. The following main conclusions are reached.

1. Intensities of OI $\lambda 1356 \AA$ emission estimated from the precipitation of magnetospheric electrons observed near Ganymede are inadequate to account for the observational diffuse auroral intensities $\leq 100 R$. This is in agreement with conclusions reached in earlier works (Eviatar et al., 2001b). Some acceleration mechanism is suggested to energize the magnetospheric electrons.
2. The use of a Maxwellian distribution to simulate the heating of electrons by electrostatic waves can provide a diffuse auroral intensity of about $70 R$ corresponding to a temperature of $150 \mathrm{eV}$.

3. The use of a kappa distribution in the case of $\kappa=2$ having a characteristic energy of $100 \mathrm{eV}$ yields an auroral intensity of about $100 R$. However, for a distribution with $\kappa=1$ intensities of similar magnitude can be obtained for a characteristic energy of $50 \mathrm{eV}$.

4. The contribution from the direct excitation of atomic oxygen is about $1 \%$, and the cascading contribution from atomic oxygen is about $2 \%$ of the total estimated intensity.

5. The potential findings of the present study may be relevant for the present JUNO and future JUICE missions to Jupiter. These missions will provide new data on electron densities, electron temperature and whistler mode amplitudes in the magnetosphere of Jupiter near Ganymede.

\section{Data availability}

We have taken data from various journal articles, whose references are given in the text of the paper. 


\section{Appendix A: Analytical yield spectrum}

Green and colleagues (Green and Singhal, 1979; Singhal et al., 1980; Singhal and Green, 1981) studied the energy degradation of monoenergetic electrons in planetary atmospheric gases using a Monte Carlo technique. A function which they have called "yield spectra" is obtained from the Monte Carlo simulation. Two-, three-, four-, and five-dimensional yield spectra are defined as follows:

Two-dimensional yield spectra $U\left(E, E_{o}\right)$ are defined as

$U\left(E, E_{o}\right)=\frac{N(E)}{\Delta E}(\mathrm{eV})^{-1}$,

where $N(E)$ is the number of electrons in the bin centered at $E$ which result after the incident electron of energy $E_{o}$ and all its secondaries, tertiaries, etc., have been completely degraded in energy. Similarly 3 -D yield spectra $U\left(E, Z, E_{o}\right)$ are defined as

$U\left(E, Z, E_{o}\right)=\frac{N(E, Z)}{\Delta E \Delta Z}(\mathrm{eV})^{-1}\left(\mathrm{gm} \mathrm{cm}^{-2}\right)^{-1}$.

Here, $N(E, Z)$ is the total number of inelastic collisions that exist in the spatial interval $\Delta Z$ around $Z$ and in the energy interval $\Delta E$ centered at $E . Z$ is the longitudinal distance along the $Z$ axis, scaled by an effective range $R\left(E_{o}\right)$. The numerical yield spectral function generated by Monte Carlo simulation is represented analytically.
Two-dimensional YS is parameterized in the form

$U_{a}\left(E, E_{k}\right)=C_{o}+C_{1} \chi+C_{2} \chi^{2}$,

where $C_{o}, C_{1}$ and $C_{2}$ are external parameters, and

$\chi=\frac{E_{k}^{\Omega}}{E+L}$,

where $L=1 \mathrm{eV}, \Omega=0.585, E_{k}$ is incident electron energy in kiloelectronvolt, and $E$ is in electron volts. The 3-D YS is represented in the form

$U_{a}\left(E, Z, E_{k}\right)=\sum_{i=0}^{2} \frac{A_{i}^{\prime}}{R^{3}} \chi^{i} G_{i}(Z)$,

where each $G_{i}$ is a micro-plume of the form

$G_{i},=\exp \left[-\beta_{i}^{2} Z^{2}+\gamma_{i} Z\right]$.

The 3-D YS is constrained to reduce to 2-D YS upon integration over $Z$. It is found that various parameters appearing in Eqs. (A5) and (A6) are not too different from gas to gas when distance is expressed in gram per centimeter square. Thus, AYS has a universal character. 
Competing interests. The authors declare that they have no conflict of interest.

Acknowledgements. This work was supported by the Planetary Sciences and Exploration Programme (PLANEX), the Indian Space Research Organization (ISRO) and PRL, Ahmedabad, under the sanctioned project scheme. Calculations reported in the present work were carried out at the Computer Centre, Banaras Hindu University.

The topical editor, C. Owen, thanks two anonymous referees for help in evaluating this paper.

\section{References}

Acuna, M. H., Behannon, K. W., and Connerney, J. E. P.: Jupiter's magnetic field and magnetosphere, in: Physics of the Jovian magnetosphere, edited by: Dessler, A., 1-50, Cambridge Univ. Press, New York, USA, 1983.

Bates, D. R.: Some problems concerning the terrestrial atmosphere above $100 \mathrm{~km}$ level, P. R. Soc. A, 253, 451-462, 1959.

Calvin, W. M. and Spencer, J. R.: Identification of $\mathrm{O}_{2}$ on Ganymede, Bull. Am. Astron. Soc., 26, 1159, 1994.

Calvin, W. M., Johnson, R. E., and Spencer, J. R.: $\mathrm{O}_{2}$ on Ganymede spectral characteristics and plasma formation mechanism, Geophys Res Lett., 23, 673-676, 1996.

Chang, H. C.: Cyclotron resonating scattering of energetic electrons by electromagnetic waves in the magnetosphere, Tech. Rep E414-1, Space telecommun and Radiosci Lab, Stanford Univ, Stanford, CA, USA, 1983.

Connerney, J. E. P., Acuna, M. H., and Ness, N. F.: Modeling the Jovian current sheet and inner magnetosphere, J. Geophys. Res., 86, 8370-8384, 1981.

Connerney, J. E. P., Acuna, M. H., and Ness, N. F.: Voyager 1 assessment of Jupiter's planetary magnetic field, J. Geophys. Res., 87, 3623-3627, 1982.

Connerney, J. E. P., Acuna, M. H., Ness, N. F., and Satoh, T.: New models of Jupiter's magnetic field constrained by the Io flux tube footprint, J. Geophys. Res., 103, 11929-11939, 1998.

Coroniti, F. V., Scarf, F. L., Kennel, C. F., and Kurth, W. S.: Analysis of chorus emissions at Jupiter, J. Geophys Res., 89, 3801-3820, 1984.

Divine, N. and Garrett, H. B.: Charged particle distributions in Jupiter's magnetosphere, J. Geophys. Res., 88, 6889-6903, 1983.

Erdman, P. W. and Zipf, E. C.: Excitation of the OI (3s 5so $-3 p^{5}$; $\lambda 7774 \AA$ ) multiplet by electron impact on $\mathrm{O}_{2}$, J. Chem. Phys., 87, 4540-4545, 1987.

Eviatar, A., Vasyliunas, V. M., and Gurnett, D. A.: The ionosphere of Ganymede, Planet. Space Sci., 49, 327-336, 2001 a.

Eviatar, A., Strobel, D. F., Wolven, B. C., Feldman, P. D., McGrath, M. A., and Williams, D. J.: Excitation of the Ganymede ultraviolet aurora, Astrophys. J., 555, 1013-1019, 2001 b.

Feldman, P. D., McGrath, M. A., Strobel, D. F., Moos, H. W., Retherford K. D., and Wolven, B. C.: HST/STIS ultraviolet imaging of polar aurora on Ganymede, Astrophys. J., 535, 1085-1090, 2000.

Frank, L. A., Paterson W. R., and Ackerson, K. L.: Low-energy electrons measurements at Ganymede with the Galileo spacecraft:
Probes of the magnetic topology, Geophys. Res. Lett., 24, 21592162, 1997.

Glauert, S. A. and Horne, R. B.: Calculation of pitch angle and energy diffusion coefficient with the PADIE code, J.Geophys, Res., 110, A04206, doi:10.1029/2004JA010851, 2005.

Green, A. E. S. and Singhal, R. P.: Microplume model of spatial yield spectra, Geophys. Res. Lett., 6, 625-628, 1979.

Grundy, W. M., Buratti, B. J., Cheng, A. F., Emery, J. P., Lunsford, A., McKinnon, W. B., Moore, J. M., Newman, S. F., Olkin, C. B., Reuter, D. C., Schenk, P. M., Spencer, J. R., Stern, S. A., Throop, H. B., Weaver, H. A., and the New Horizens team: New Horizens mapping of Europa and Ganymede, Science, 318, 234 237, 2007.

Gurnett, D. A. and Scarf, F. L.: Plasma waves in the Jovian magnetosphere, in: Physics of the Jovian Magnetosphere, edited by: Dessler, A. J., 285-316, Cambridge Univ. Press, Cambridge, UK, 1983.

Gurnett, D. A., Kurth, W. S., and Scarf, F. L.: Plasma wave observations near Jupiter: Initial results from Voyager 2, Science, 206, 987-991, 1979.

Gurnett, D. A., Kurth, W. S., Roux, A., Bolton, S. J., and Kennel, C. F.: Evidence for a magnetosphere at Ganymede from plasmawave observations by the Galileo spacecraft, Nature, 384, 535537, 1996.

Hall, D. T., Feldman, P. D., McGrath, M. A., and Strobel, D. F.: The Far-Ultaviolet oxygen airglow of Europa and Ganymede, Astrophys. J., 499, 475-481, 1998.

Itikawa, Y., Ichimura, A., Onda, K., Sakimoto, K., Takayanagi, K., Hatano, Y., Hayashi, M., Nishimura, H., and Tsurubuchi, S.: Cross-sections for collisions of electrons and photons with oxygen molecules, J. Chem. Phys. Ref. Data, 18, 23-42, 1989.

Jackman, C. H., Garvey, R. H., and Green, A. E. S.: Electron impact on atmospheric gases I updated cross-section, J. Geophys Res., 82, 5081-5090, 1977.

Kennel, C. F.: Consequences of a magnetospheric plasma, Rev. Geophys, 7, 379-419, 1969.

Kennel, C. F. and Petschek, H. E.: Limit on stably trapped particles fluxes, J. Geophys. Res., 71, 1-28, 1966.

Kivelson, M. G., Khurana, K. K., Russell, C. T., Walker, R. J., Warnecke, J., Coroniti, F. V., Polanskey, C., Southwood, D. J., and Schubert, G.: Discovery of Ganymede's magnetic field by the Galileo spacecraft, Nature, 384, 537-541, 1996.

Kivelson, M. G., Khurana, K. K., Coroniti, F. V., Joy, S., Russell, C. T., Walker, R. J., Warnecke, J., Bennett, L., and Polanskey, C.: The magnetic field and magnetosphere of Ganymede, Geophys. Res. Lett., 24, 2155-2158, 1997.

Kivelson, M. G., Khurana, K. K., and Volwerk, M.: The permanent and inductive moment of Ganymede, Icarus, 157, 507-522, 1998.

Kurth, W. S, Gurnett, D. A., Roux, A., and Bolton, S. J.: Ganymede: A new radio source, Geophys. Res. Lett., 24, 2167-2170, 1997.

Lavrukhin, A. S. and Alexeev, I. I.: Aurora at high latitude of Ganymede, 2015, Astronomy Lett., 41, 687-692, 2015.

Lyons, L. R.: Electron diffusion driven by magnetospheric electrostatic waves, J. Geophys Res., 79, 575-580, 1974.

Lyons, L. R., Thorne, R. M., and Kennel, C. F.: Pitch angle diffusion of radiation belt electrons within the plasmasphere, J. Geophys. Res., 77, 3455-3474, 1972. 
Mantas, G. P.: Theory of photoelectron thermalization and transport in the ionosphere, Planet. Space Sci., 23, 337-354, 1975.

McGrath, M. A., Jia, X., Retherford, K., Feldman, P. D., Strobel, D. F., and Saur, J.: Aurora on Ganymede, J. Geophys. Res., 118, 2043-2054, 2013.

Mead, G. D.: Pioneer 10 mission: Jupiter encounter, J. Geophys. Res., 79, 3487-3487, 1974.

Menietti, J. D., Horne, R. B., Gurnett, D. A., Hospodarsky, G. B., Piker, C. W., and Groene, J. B.: A survey of Galileo plasma wave instrument observations of Jovian whistler-mode chorus, Ann. Geophys., 26, 1819-1828, doi:10.5194/angeo-26-18192008, 2008.

Ni, B., Liang, J., Thorne, R. M., Angelopoulos, V., Horne, R. B., Kubyshkina, M., Spanswick, E., Donovan, E. F., and Lummerzheim, D.: Efficient diffuse auroral electron scattering by electrostatic electron cyclotron harmonic waves in outer magnetosphere: A detailed case study, J. Geophys. Res., 117, A01218, doi:10:1029/2011 JA017095, 2012.

Orlova, K. G. and Shprits, Y. Y.: On the bounce-averaged of scattering rates and the calculations of bounce period, Phys. Plasmas, 18, 092904, doi:10.1063/1.3638137, 2011.

Paranicas, C., Paterson, W. R., Cheng, A. F., Mauk, B. H., McEntire, R. W., Frank, L. A., and Williams, D. J.: Energetic particle observations near Ganymede, J. Geophys. Res., 104, 17459-17469, 1999.

Scarf, F. L., Gurnett, D. A., and Kurth, W. S.: Jupiter plasma wave observations: An initial Voyager 1 overview, Science, 204, 991995, 1979.

Singhal, R. P. and Green, A. E. S.: Spatial aspects of electron energy degradation in atomic oxygen, J. Geophys. Res., 86, 4776-4780, 1981.

Singhal, R. P. and Tripathi, A. K.: Study of whistler mode instability in Saturn's magnetosphere, Ann. Geophys., 24, 1705-1712, doi:10.5194/angeo-24-1705-2006, 2006.

Singhal, R. P., Jackman, C. H., and Green, A. E. S.: Spatial aspects of low and medium energy electron degradation in $\mathrm{N}_{2}$, J. Geophys. Res., 85, 1246-1254, 1980.

Singhal, R. P., Tripathi, A. K., Halder, S., and Singh II, O. N.: Diffuse aurora on Ganymede driven by electrostatic waves, Astrophys. J., 832, 172-180, doi:10.3847/0004-637X/832/2/172, 2016.

Spencer, J. R., Calvin, W. M., and Persoon, M. J.: CCD spectra of the Galilean satellites: Molecular oxygen on Ganymede, J. Geophys. Res., 100, 19049-19056, 1995.
Stone, R. G., Pedersen, B. M., Harvey, C. C., Canu, P., CornilleauWehrlin, N., Desch, M. D., de Villedary, C., Fainberg, J., Farrell, W. M., Goetz, K., Hess, R. A., Hoang, S., Kaiser, M. L., Kellogg, P. J., Lecacheux, A., Lin, N., Macdowall, R. J, Manning, R., Meetre, C. A., Meyer-Vernet, N., Moncuquet, M., Osherovich, V., Reiner, M. J., Tekle, A., Thiessen, J., and Zarka, P.: Ulysses radio and plasma wave observations in the Jupiter environment, Science, 257, 1527-1531, 1992.

Summers, D. and Thorne, R. M.: The modified plasma dispersions function, Phys. Fluids B, 3, 1835-1847, 1991.

Summers, D., Xue, S., and Thorne, R. M.: Calculation of the dielectric tensor for a generalized Lorentzian (kappa) distribution function, Phys. Plasmas., 1, 2012-2025, 1994.

Susanna, M., Ferenc, V., William, M., and Gerald, S.: Numerical simulations of the orbits of the Galilean satellites, Icarus, 159, 500-504, 2002.

Swift, D. W.: Particle acceleration by electrostatic waves, J. Geophys. Res., 75, 6324-6328, 1970.

Tripathi, A. K., Singhal, R. P., Singh, K. P., and Singh II, O. N.: Pitch angle diffusion by whistler mode waves in the Jovian magnetosphere and diffuse auroral precipitation, Icarus, 225, 424431, 2013.

Tripathi, A. K., Singhal, R. P., Singh, K. P., and Singh II, O. N.: Whistler mode instability and pitch-angle diffusion near Ganymede, Planet. Space. Sci., 92, 150-156, 2014.

Wells, W. C., Borst, W. L., and Zipf, E. C.: Absolute cross-section for the production of $\mathrm{O}\left({ }^{5} s^{\circ}\right)$ by electron impact dissociation of $\mathrm{O}_{2}$, Chem. Phys. Lett., 12, 288-290, 1971.

Williams, D. J. and Mauk, B.: Pitch-angle diffusion at Jupiter's moon Ganymede, J. Geophys. Res., 102, 24283-24287, 1997.

Williams, D. J., McEntire, R. W., Jaskulek, S., and Wilken, B.: The Galileo energetic particles detector, Space Sci. Rev., 60, 385412, 1998.

Williams, D. J., Mauk, B., and McEntire, R. W.: Properties of Ganymede's magnetosphere as revealed by energetic particle observations, J. Geophys. Res., 103, 17523-17534 1997a.

William, D. J., Mauk, B., and McEntire, R. W.: Trapped electrons in Ganymede's magnetic field, Geophys Res. Lett., 24, 2953-2956, 1997b. 